\title{
New Directions Toward Nanophysics Experiments in STEM
}

Mathieu Kociak ${ }^{1}$, Jean-Denis Blazit ${ }^{1}$, Laura Bocher ${ }^{1}$, Nathalie Brun ${ }^{1}$, Christian Colliex ${ }^{1}$, Marta de Frutos $^{1}$, Alexandre Gloter ${ }^{1}$, Xiaoyan Li $^{1}$, Marcel Tencé ${ }^{1}$, Luiz H. G. Tizei ${ }^{1}$, Michael Walls ${ }^{1}$, Alberto Zobelli $^{1}$ and Odile Stéphan ${ }^{1}$

${ }^{1 .}$ Laboratoire de Physique des Solides, CNRS, Université Paris Sud, Orsay, France.

Recently the electron microscopy community has enjoyed great successes in STEM aberration correction and electron-beam monochromation for EELS spectroscopy. More exciting applications will emerge from the combination of such extraordinary instrumental progress with conceptual and methodological advances and complementary instrumental developments. At Orsay, some progress has been made in this direction. Notably, the use of EELS detectors with single-electron sensitivity has been demonstrated to provide EELS spectra with enough signal-to-noise ratio to evaluate doping and chemical changes of the order of a few percent while maintaining atomic resolution. Characterizing the energy stability has been shown to be crucial when measuring minute spectral shifts in connection with the quantitative estimation of electron densities and 2D gas property changes at interfaces in oxide-based nanodevices [1]. In the case of highly sensitive materials, new scanning acquisition strategies for limiting the electron dose while optimizing the signal detection, in combination with advanced data processing, are opening exciting perspectives [2]. Novel ideas have emerged for exploiting fast electron beams, including combining them with beams of photons, to provide a high-spatial-resolution alternative to more conventional optical techniques. Recent experiments combining EELS and CL have shown the interest of such an approach for testing conventional macroscopic concepts such as extinction, absorption, and scattering and adapting them to describe optical phenomena at the nanoscale $[3,4,5]$. New developments have also been realized for exploring the intimate link in 2D materials between the crystal structure (including defects) and the optical properties as revealed by nano-CL [6]. Measuring the photon statistics from isolated emission centres has also been demonstrated, opening new routes into the field of quantum nano-optics [7].

Implementing these developments in a next-generation, highly monochromatized STEM with ultra-high energy resolution (down to 5-10 meV) in EELS and equipped with a light injection/detection system and a low-temperature stage is the objective of the CHROMATEM project lead by the Orsay team. The motivations for such a setup are numerous [8].

1) Monochromation obviously improves the energy resolution in EELS but more importantly it provides access to new spectral signatures in the low-loss range of primary interest in condensed matter physics and in the field of nano-optics. For instance, in the case of transition-metal oxides, various degrees of freedom (spin, charge...) are present and playing a role. EELS is bound to become a privileged technique for probing almost all the low-energy excitations related to the presence and interplay of all these degrees of freedom: these are for example the rather localized d-d transitions, charge transfer excitations or excitations related to the long-range order of the system (plasmons, magnons, orbitons, phonons ...).

2) There is a need for combined photon and electron spectroscopic measurements. Indeed, different spectroscopic techniques provoke a given excitation in different ways, furnishing related but different information. Combining luminescence and/or scattering spectroscopies (CL) with absorption/extinction 
spectroscopy (EELS) is of primary importance in plasmonics (as mentioned above) but also for probing the physics of excitonic excitations and their relaxation processes, for example. Beyond the comparison between different spectroscopy techniques, coincidence detection and photon/electron beam synchronization might be also accessible in the context of CHROMATEM, to time-correlate EELS and CL or to perform pump-probe experiments.

3) Finally, high-precision temperature control, as is standard in optical luminescence or synchrotron-based spectroscopic methods, would considerably widen the application range of high resolution spectroscopy in the TEM. The most obvious advantages are an enhanced visibility of the spectral signatures (this is crucial for excitons), the ability to probe the temperature dependence of an excitation (which might be the signature of peculiar electronic structure features, as in the case of the exciton line intensities in confined semiconductors for example) and most obviously, the access to phase transitions (the metal-insulator transition in oxides is an archetypal example). In this context, additional external stimuli are also foreseen (light illumination, electrical biasing etc...).

The CHROMATEM microscope, a side-entry Nion high energy resolution monochromated EELS (HERMES) [9] incorporating an Attolight Mönch 4107 light detection/injection system, is under testing (and already providing sub-10 meV spectral resolution) at the time of submitting this abstract. This talk will therefore summarise some of the most recent results in STEM EELS and CL obtained at Orsay, including any available results obtained on the CHROMATEM microscope.

\section{References :}

[1] M. Marinova et al, Nano Lett. 15 (2015), p. 2533.

[2] See A. Zobelli et al, this Conference.

[3] A. Losquin et al, Nano Lett. 15 (2015), p.1229.

[4] N Kawasaki et al, ACS Photonics 3 (2016), p. 1654.

[5] Hugo Lourenço-Martins et al, Nature Physics (2018), doi:10.1038/s41567-017-0023-6.

[6] R. Bourrellier et al, ACS Photonics 1 (2014), p. 857.

[7] R. Bourrellier et al, Nanolett. 16 (2016), p. 4317.

[8] M. Kociak, A Gloter, O. Stéphan, Ultramicroscopy 180 (2017), p. 81.

[9] See M.T. Hotz et al, this Conference.

[10] This work has received support from the National Agency for Research under the program of future investment TEMPOS- CHROMATEM with the Reference No. ANR-10-EQPX- 50. 